\title{
Safety and Immunogenicity of RTS,S/AS01 Malaria Vaccine in Infants and Children with WHO Stage 1 or 2 HIV Disease: A Randomized, Double-Blind, Controlled Trial
}

Lucas Otieno $M D^{a}$, Martina Oneko $M D^{b}$, Walter Otieno PhD ${ }^{a}$, Joseph Abuodha MBChB ${ }^{b}$, Emmanuel Owino MSc ${ }^{a}$, Chris Odero PGD PH ${ }^{b}$, Yolanda Guerra Mendoza MD ${ }^{c}$, Ben Andagalu MSc ${ }^{a}$, Norbert

Awino MSc ${ }^{b}$, Karen Ivinson BSc ${ }^{d}$, Dirk Heerwegh PhD ${ }^{c}$, Nekoye Otsyula MSc ${ }^{a}$, Maria Oziemkowska $M P H^{b}$, Effua Abigail Usuf PhD ${ }^{c}$, Allan Otieno $M D^{a}$, Kephas Otieno MPH ${ }^{b}$, Didier Leboulleux $M D^{d}$, Amanda Leach MRCPCH ${ }^{c}$, Janet Oyieko MD ${ }^{a}$, Laurence Slutsker $M^{b}$, Marc Lievens $M S c^{c}$, Jessica Cowden $M D^{a}$, Didier Lapierre $M D^{c}$, Simon Kariuki $P h D^{b}$, Bernhards Ogutu PhD ${ }^{a}$, Johan Vekemans PhD $D^{c}$ Mary J. Hamel MD

${ }^{a}$ KEMRI-Walter Reed Project, Kombewa, Kenya

${ }^{\mathrm{b}} \mathrm{KEMRI/CDC}$ Research and Public Health Collaboration, Kisumu, Kenya

${ }^{\mathrm{c}}$ GSK Vaccines, Wavre, Belgium

dPATH Malaria Vaccine Initiative, Washington D.C., USA

Corresponding Author: Dr Lucas Otieno, KEMRI-Walter Reed Project, Kombewa, Kenya; Lucas.Tina@usamru-k.org; Tel: + 254720597654

\section{SUMMARY}

BACKGROUND Malaria remains a major public health concern. RTS,S/AS01 malaria vaccine was reviewed by the European Medicines Agency and received a positive scientific opinion; the World Health Organization subsequently recommended pilot implementation in sub-Saharan African countries. Because malaria and HIV overlap geographically, HIV infected children will be considered for RTS,S/AS01 vaccination. We evaluated the safety of RTS,S/AS01 in HIV-infected children enrolled at two sites in Western Kenya.

METHODS Children aged 6 weeks to 17 months with WHO stage 1 or 2 HIV disease were randomized 1:1 to receive 3 doses RTS,S/AS01 or rabies vaccine, administered monthly. Antiretrovirals and daily cotrimoxazole were provided. Serious adverse events (SAEs), reactogenicity, HIV disease progression, immunogenicity, and vaccine efficacy against clinical malaria were measured during 14 months followup. ClinicalTrials.gov, number NCT01148459 
FINDINGS One hundred-seventy-seven of 200 children enrolled (89\%) completed the study. SAEs were experienced by $41.4 \%$ (95\%CI: 31.6-51.8) and 36.6\% (95\%CI: 27.3-46.8) in RTS,S/AS01 and rabies recipients, respectively; $20.2 \%$ (95\%CI: $12.8-29.5)$ and $11.9 \%$ (95\%CI:6.3-19.8) occurred within 30 days post-vaccination mainly due to pneumonia and febrile convulsions. Five (5.1\%, 95\% CI: 1.7-11.4) RTS,S/AS01 recipients and 4 (4.0\%, 95\%CI:1-1-9.8) rabies vaccine recipients died. One related SAE was reported: a febrile convulsion in an RTS,S/AS01 recipient. No difference in HIV disease progression (CD4+ T-cell, HIV viral load and WHO clinical staging) was seen between study arms. A non-significant trend for a higher median viral load in the RTS,S/AS01 arm was observed. RTS,S/AS01 recipients had an anti-CS antibody geometric mean titer (EU/mL) of 329.2\% (95\%CI: 260.6-415.8) at 1 month post-dose 3 and a strong immune response against hepatitis B.

INTERPRETATION RTS, S/AS01 was safe and immunogenic when administered to children with WHO clinical stage 1 or 2 HIV disease in the context of high ART and CTX use. Children with HIV disease could be included in future RTS,S/AS01vaccination programs.

FUNDING GlaxoSmithKline Biologicals SA and PATH Malaria Vaccine Initiative

\section{DISCLAIMER}

The opinions and assertions herein are the views of the authors and not those of KEMRI, the Department of Defense, Centers for Disease Control and Prevention or US Government. 


\section{INTRODUCTION}

Malaria remains an important global public health problem and a major cause of childhood morbidity and mortality. This is especially the case in sub-Saharan Africa, where more than $88 \%$ of annual malaria episodes occur ${ }^{1}$. A malaria vaccine would play an important role in reducing malaria morbidity and mortality ${ }^{2}$. The geography of malaria and HIV overlap considerably in sub-Saharan Africa ${ }^{1,3}$, therefore it would be important to assess the safety of a malaria vaccine in those who are HIV-infected and who may be eligible for vaccination.

The RTS,S/AS01 malaria vaccine has been evaluated for safety and efficacy in a large phase III, multisite trial conducted in seven African countries. The vaccine efficacy (VE) against clinical malaria over the first 12 months was 51.3\% in children aged 5-17 months and 32.9\% in young infants aged 6-12 weeks. Over the study duration, (median participant follow up period of 48 months in children and 38 in infants), VE in children was $39.0 \%$ with the booster and $26.2 \%$ without the booster while in young infants, VE was $26.79 \%$ with the booster dose and $18.2 \%$ without booster. ${ }^{4-6}$ RTS,S/AS01 was reviewed by the Committee for Medicinal Products for Human Use (CHMP) of the European Medicines Agency and received a positive scientific opinion. ${ }^{7}$ The World Health Organization (WHO) recommended pilot implementation of RTS,S/AS01 in children in 3-5 sub-Saharan African countries with moderate to high malaria transmission levels to measure feasibility, impact and safety of the vaccine when a 4 dose schedule is implemented through the routine health services. WHO will consider the results from the RTS,S/AS01 pilots in formulating recommendations for RTS,S/AS01 vaccine use. ${ }^{8}$

The RTS,S/AS01 vaccine is developed for use in the routine immunization of African children to reduce malaria morbidity and mortality. The vaccine consists of sequences of the Plasmodium falciparum circumsporozoite (CS) protein and hepatitis B surface antigen ( $\mathrm{HBsAg}$ ) with the novel proprietary adjuvant AS01 (liposome formulations of mono-phosphoryl lipid A (MPL) and QS21 immunostimulants). ${ }^{9,10}$

Given the geographic overlap in Malaria and HIV infection in some populations targeted ${ }^{11,12}$ for this vaccine in sub-Saharan Africa, the safety and immunogenicity of RTS,S/AS01 in HIV-infected children is an essential component of the overall vaccine profile. Immunization with other vaccines has been found to be safe and beneficial for children infected with HIV. Consequently, WHO guidelines recommend including children with HIV in national immunization programs. For non-live vaccines, HIV infection has not been shown to be associated with an increased risk while for live attenuated vaccines like measles, yellow fever and BCG, the degree of immunosuppression is considered prior to vaccination. ${ }^{\mathbf{1 3}}$ The 
primary objective of this trial was to evaluate the safety of RTS, S/AS01 in young children known to be HIV infected. Other objectives included an assessment of vaccine immunogenicity by measuring antibody responses to circumsporozoite and hepatitis B surface antigens, HIV disease progression, exploratory evaluation of vaccine efficacy, and growth monitoring.

\section{METHODS}

\section{Study design}

This randomized, controlled, double blind (observer blinded) trial conducted from 30 July 2010 to 24 May 2013 aimed to evaluate the safety and immunogenicity of RTS,S/AS01 when administered on a 0, 1, 2-month schedule to HIV-infected children (supplement Figure 1, page 1). The trial protocol was approved by the Scientific and Ethics Committees of the Kenya Medical Research Institute (KEMRI), the Walter Reed Army Institute of Research (WRAIR), the US Centers for Disease Control and Prevention (CDC) and the Western Institutional Review Board (WIRB). Written informed consent was obtained from the participants' parent(s) or guardian(s). The trial was undertaken in accordance with the principles of Good Clinical Practice.

\section{Study site and participants}

Two-hundred children aged 6 weeks to 17 months (inclusive) at first vaccination were enrolled at two study sites in Western Kenya, KEMRI/WRAIR, Kisumu and KEMRI/CDC, Siaya. The sites are located in an area with high malaria prevalence (38\% in children $0-14$ years) ${ }^{14}$ and vertical transmission rates of HIV (between $7-15 \%$ in the context of increased uptake of PMCT) ${ }^{15}{ }^{16}$. Infants and children known to be HIV infected (documented positive DNA PCR) were included, whether or not they were on antiretroviral medication. All children received an insecticide treated bednet at screening. Exclusion criteria were moderate or severe illness, HIV stage III or IV disease (WHO pediatric AIDS clinical staging) at enrollment, haemoglobin $<5 \mathrm{~g} / \mathrm{dL}$, neutropenia (WBC (White blood cell count) $<1 \times 10^{3} / \mu \mathrm{L}$ ), severe thrombocytopenia (platelet count $<25 \times 10^{3} / \mu \mathrm{L}$ ), elevated liver enzymes (ALT (alanine aminotransferase) $>5 \mathrm{x}$ upper limit of normal range) and renal dysfunction (creatinine $>3 \mathrm{x}$ upper limit of normal range). Children born at $<36$ weeks or $>42$ weeks, same sex twins and children born with major congenital abnormalities were excluded.

\section{Randomization and masking}

Eligible participants were randomly assigned (1:1 ratio) into two study arms to receive either RTS,S/AS01 malaria candidate vaccine (RTS,S/AS01 arm) or Vero Rab Rabies vaccine from Sanofi- 
Pasteur (control arm). The treatment allocation was performed using a central randomization system on the internet (MATEX), a program developed for use in SAS® (Cary, NC, USA) by GSK Biologicals. Randomization was balanced to ensure that both groups were comparable in terms of age (6 weeks to 4 months and 5 to 17 months) and CD4 percentage ( $<10 \%, 10-14 \%, 15-19 \%, \geq 20 \%)$ with an equivalent number of children enrolled in both study groups.

Data pertaining to RTS,S/AS01 or the rabies vaccine were collected in a double blinded (observer blinded) manner with the vaccine recipient and their parent(s)/Legally Acceptable Representative(s), the sponsor as well as those responsible for the evaluation of safety and immunogenicity endpoints all unaware of the treatment arm administered to a particular subject. The only study staff aware of vaccine assignment were those responsible for the preparation and administration of vaccines; these staff were considered unblinded and played no other role in the study.

\section{Study procedures}

Safety surveillance

Safety surveillance was conducted over 14 months from the time of dose 1. Serious adverse events (SAE) were collected from the time of first vaccination by passive surveillance, and included all hospitalizations, life threatening events, events resulting in death or disability, all seizures within 30 days of vaccination, and immune mediated disorders. Seizures that occurred within seven days after vaccination were analyzed according to Brighton Collaboration guidelines. ${ }^{17}$ Verbal autopsies were conducted on deaths that occurred outside study facilities. ${ }^{18}$

Solicited local adverse events (AE), (pain, swelling or redness at the injection site), and general AEs, (axillary temperature $\geq 37 \cdot 5^{\circ} \mathrm{C}$, drowsiness, irritability or loss of appetite), were collected during seven days after vaccination. All unsolicited AEs were collected during 30 days after vaccination.

Safety laboratory tests, including Complete Blood Count, Creatinine and Alanine-Aminotransferase (ALT), were scheduled seven days post-dose 1, 1 month post-dose 3 and at study conclusion. These laboratory values were evaluated using an adapted WHO Toxicity Grading Scale (WHO 2003, supplement table 1, page 3).

HIV disease progression was monitored at specified time points by WHO HIV clinical staging, CD4+ Tcell, and HIV viral load (VL). Antiretroviral treatment (ART) combination regimens (Abacavir or 
Zidovudine, Lamivudine, Nevirapine or Lopinavir/Ritonavir) and daily cotrimoxazole (CTX) was provided to all participants for the prevention of opportunistic infections.

Immunogenicity

Blood samples were collected at baseline, 1 month post-dose 3 and study end to assess immunogenicity. Antibody responses to the central repeat region of the circumsporozoite (CS) antigen were measured by ELISA $^{19}$ and reported by Geometric Mean Titers (GMT) and percentage seropositivity with an anti-CS cut-off value of $\geq 0.5 \mathrm{EU} / \mathrm{mL}$. Antibody responses against Hepatitis B virus surface antigen ( $\mathrm{HbsAg})$ were also measured by ELISA and reported by GMT and expressed in $\mathrm{mIU} / \mathrm{mL}$ with a cut-off for seroprotection of $10 \mathrm{mIU} / \mathrm{mL}$.

Malaria surveillance

Passive surveillance for malaria was carried out from dose 1 to study conclusion. Participants were encouraged to seek care at study clinics for any illness, and transportation was facilitated. Participants who presented with a temperature $\geq 37.5{ }^{\circ} \mathrm{C}$ or reported fever within the prior 24 hours had a blood sample taken for detection and quantification of parasites by microscopy. At 14 months post-dose 1, a blood slide was made to measure malaria parasite prevalence.

\section{Outcomes}

The primary outcome was occurrence of SAEs from the time of first vaccination until 14 months postdose 1 . The secondary outcomes of the study included safety, immunogenicity, malaria monitoring, HIV progression and growth monitoring. Safety assessment included general and local solicited symptoms, unsolicited symptoms and non-malaria-related SAEs. Immunogenicity involved measurement of anti-CS antibody titers, and anti-HBs antibody titers. Clinical malaria incidence was measured throughout the trial and malaria parasitaemia prevalence was measured at 12 months post-dose 3 . HIV disease progression was determined based on VL, CD4+ \% and absolute cell counts and WHO HIV clinical classification. Growth monitoring was assessed by anthropometric measurements.

\section{Statistical analysis}

The sample size was based on the primary endpoint of the trial which was an assessment of vaccine safety. The trial had a total of 200 participants, randomized 1:1 to each arm; for an event occurring with a frequency of 1/100 controls there was $80 \%$ power to detect an eight fold increase in proportion of SAEs between the RTS,S/AS01 arm versus the rabies vaccine arm ( 2 sided Fisher exact test; alpha=0.05). The statistical analyses were done using SAS Drug Development (SDD) version 3.5. 
All safety analyses were performed on the Intention-to-Treat (ITT) population. SAEs and unsolicited AEs were classified according to the Medical Dictionary for Regulatory Activities (MedDRA). The proportion of subjects with SAEs or AEs within 30 days post vaccination was tabulated with exact $95 \%$ CI. Viral load, CD4+ T-cell and WHO clinical staging were tabulated by study arm for each time-point.

Primary analysis of immunogenicity was based on the per-protocol population (ATP population for immunogenicity). Anti-CS antibody titers were evaluated post-dose 3 by sero-positivity rates and GMTs at each blood sampling time point. As an exploratory endpoint, efficacy against clinical and severe malaria was evaluated in the ATP population, which included subjects who received all vaccinations within specified intervals and who contributed time at risk in the follow-up period starting 14 days postdose 3 , and in the ITT population where time at risk started on the day of first vaccination.

VE against clinical malaria was estimated as (1 - Hazard Ratio) using Cox models (first or only episodes) and Negative binomial models (All episodes). The frequency of subjects with severe malaria and malaria hospitalization was presented according to the treatment arm. Exploratory models (ANCOVA) were developed to further analyze the change from baseline in HIV VL and CD4 \%. ${ }^{20}$

\section{Case definitions for clinical and severe malaria}

The primary case definition of clinical malaria was an illness in a child brought to a study facility with an axillary temperature of $\geq 37 \cdot 5^{\circ} \mathrm{C}$ and $P$. falciparum asexual parasitaemia $>2500$ parasites $/ \mu \mathrm{L}$. The primary case definition of severe malaria was $P$. falciparum $>2500$ parasites per $\mu \mathrm{L}$ and with one or more marker of disease severity and without a diagnosis of co-morbidity. The secondary case definitions of clinical and severe malaria can be found in the supplement (supplement table 2, page 3).

\section{Study oversight and role of the funding source}

The study was sponsored by GSK Biologicals SA (GSK), and funded by both GSK and PATH Malaria Vaccine Initiative (MVI). The study investigators designed and developed the study in collaboration with GSK with input from MVI. Analysis was performed by DH and ML according to the analysis plan; all authors reviewed the data and had the final responsibility for the decision to submit for publication. The corresponding author and writing team had access to the data in the study and had final responsibility for 
the decision to submit for publication. This study is registered with ClinicalTrials.gov, number NCT NCT01148459

\section{RESULTS}

\section{Baseline characteristics}

Two-hundred-seventy-five HIV-infected children were screened, and 200 enrolled, 99 into the RTS,S/AS01 arm and 101 into the rabies vaccine arm (figure 1). One-hundred-twenty-three (61.5\%) participants were enrolled in Kisumu and 77 (38.5\%) in Siaya. One-hundred-seventy-seven (89\%) completed 14 months follow-up.

Baseline characteristics were similar between the two study arms (table 1). The mean age at first vaccination was 10 months in the RTS,S/AS01 arm and 9.5 months in the rabies vaccine arm. In both the RTS,S/AS01 and rabies vaccine arms, the ratio of younger (6 weeks to 4 months) to older participants (5 months to 17 months) enrolled was $20 \%$ to $80 \%$ with males accounting for $42 \%$ in the RTS,S/AS0 1 arm and $55 \%$ in the rabies vaccine arm.

\section{Safety}

Overall, the percentage of children experiencing at least one SAE over 14 months of follow up was similar between RTS,S/AS01 recipients $(41 \cdot 4 \%$; 95\%CI: 31.6-51.8) and rabies vaccine recipients (36.6\%; 95\%CI: 27-3-46.8). The most common SAEs between RTS,S/AS01 and Rabies vaccine recipients were: pneumonia $(23 \cdot 2 \%$; $95 \%$ CI: $15 \cdot 3-32 \cdot 8)$ vs. $(22 \cdot 8 \%$; $95 \% \mathrm{CI}: 15 \cdot 0-32 \cdot 2)$, gastroenteritis $(21 \cdot 2 \%$; 95\%CI: $13 \cdot 6-30 \cdot 6)$ vs. $(18 \cdot 8 \%$; 95\%CI: $11 \cdot 7-27 \cdot 8)$ and febrile convulsion $(10 \cdot 1 \%$; 95\%CI: 5.0$17 \cdot 8)$ vs.(12.9\%; 95\%CI:7·0-21·0). A comparison of other SAEs between the two study arms are presented in table 2.

Five children $(5 \cdot 1 \%$; $95 \%$ CI: $1 \cdot 7 \%-11 \cdot 4 \%)$ in the RTS,S/AS01 arm versus $4(4 \cdot 0 \%$; $95 \%$ CI: $1 \cdot 1 \%$ $9.8 \%$ ) in the rabies vaccine arm died (table 2 and supplement figure 2, page 1). Mortality in this HIVinfected population was associated with pneumonia, gastroenteritis, malnutrition, sepsis, Haemophilus influenzae meningitis and tuberculosis. No deaths were related to vaccination.

One SAE, a febrile convulsion that occurred one day after the third dose of RTS,S/AS01 was related to vaccination. During the 30 days following each vaccination dose, $20(20 \cdot 2 \%, 95 \% \mathrm{CI}: 12 \cdot 8-29 \cdot 5)$ recipients of RTS,S/AS01 vaccine experienced at least one SAE compared to $12(11 \cdot 9 \%, 95 \% \mathrm{CI}: 6 \cdot 3-$ 
19.8) recipients of the rabies vaccine (supplement table 3, page 4). The difference was in part explained by a numerical imbalance in the number of pneumonia events reported in 13 (13.1\%; 95\% CI: 7.2-21.4) RTS,S/AS01 recipients compared to $5(5 \%$; 95\% CI:1.6-11.2) rabies vaccine recipients (supplement table 3, page 4). This initial imbalance in pneumonia cases did not persist during the 14 month follow-up period (table 2).

The frequency of both local and generalized reactions within seven days of vaccination was higher in the RTS,S/AS01 arm compared with the rabies vaccine arm, including a higher frequency of pain at injection site $(18.1 \%)$ vs. $(6 \%)$, fever $(41.7 \%)$ vs. $(18.8 \%)$, irritability (25.3\%) vs. (10.7\%), and loss of appetite $(17.7 \%)$ vs. (8.7\%) respectively. Overall, grade 3 solicited adverse events were low in both study arms, $4 \cdot 2 \%$ (95\%CI: $2 \cdot 2-7 \cdot 2)$ and 2\% (95\%CI: 0.7-4.3) in the RTS,S/AS01 and rabies vaccine arms respectively (figure 2).

Unsolicited AEs were similar between RTS,S/AS01 and rabies vaccine recipients during the 30 days following each vaccination (supplement table 4, page 5). A grade 3 ALT elevation was observed in one subject receiving the rabies vaccine 12 months post-dose 3. No other Grade 3 or 4 biochemistry or hematology parameters were observed during the entire study.

\section{HIV disease progression}

HIV disease progression was assessed at screening, 1, 6 and 12 months post vaccine dose-3. At screening HIV-specific parameters were similar in the two study arms; the median CD4 cell percentage was (27\% (21-32) vs. 26\% (21-31), the median CD4 cell count (number of cells/ $\mu \mathrm{L}$ ) was 1995 (1325-2683) vs. 1896 (1408-2447), the median HIV viral load was 149000 (7490-750000) vs. 157000 (3630-750000) and WHO HIV clinical stage 1 disease $81.8 \%$ vs. $81.2 \%$ in the RTS,S/AS01 and rabies vaccine arm, respectively. Although CD4+ T-cell, and WHO HIV clinical stage remained similar between the study arms at each assessment, a higher median VL was seen at 1 month $(\mathrm{VL}=3125$ vs. 584$)$ and 6 months ( $\mathrm{VL}=3790$ vs.400), and to a lesser extent, at 12 months (VL= 947 vs. 400) post-dose 3 in the RTS,S/AS01 arm compared with the rabies vaccine arm, but with overlapping confidence intervals. This imbalance in VL followed a dramatic drop in the median HIV VL after the screening period, which coincided with increased uptake of ART between screening and 1 month post-dose 3 (table 3 and supplement table 5 , page 7).

At baseline, the proportion of patients with detectable HIV VL was similar between the sites for the RTS,S/AS01 arm with KEMRI/WRAIR Kisumu at 84.7\% and KEMRI/CDC Siaya at 85\% (Fisher p value $=1.000$ ) but differed for the rabies vaccine arm with a statistically non-significant (Fisher $p$ 
value $=0.1331)$ higher proportion of detectable HIV VL in Kombewa at $90.6 \%$ compared to $78.4 \%$. During subsequent visits at 1 month, 6 months and 12 months post-dose 3, the proportion of detectable HIV VL levels remained relatively higher in Kisumu compared to Siaya (supplement table 6, page 8).

Exploring this finding further by post hoc analysis, modelling the change from baseline VL demonstrated that baseline VL ( $<<0.01$ at all time points), site ( $<<0.05$ at all time points) and early CTX use at dose 1 ( $<<0.05$ at 6 and 12 months post-dose 3 ) but not vaccine group ( $>>0.1$ at all time points) were significantly associated with changes in VL over time (supplement tables 7 and 8, pages 9 and 10).

\section{Immunogenicity}

At baseline, anti-CS seropositivity was similar between study arms, at $19 \cdot 8 \%$ (95\%CI: $11 \cdot 7-30 \cdot 1)$ in the RTS,S/AS01 arm and 17.8\% (95\%CI: 9.8-28.5) in the rabies vaccine arm. Among anti-CS seropositive children, the GMT of anti-CS antibodies was low [GMT $=0 \cdot 3 \mathrm{EU} / \mathrm{mL}$ (95\%CI: 0-3-0.4)]. One month post-dose 3, 100\% (95\%CI: 95.4-100) of children in the RTS,S/AS01 arm were anti-CS seropositive, with a GMT of 329.2 EU/mL (95\%CI: 260 6-415.8). At 12 month post-dose 3, anti-CS seropositivity remained high in the RTS,S/AS01 arm at 98.6\% (95\%CI: 92.6-100) but the GMT had fallen to 18.4 EU/mL (95\%CI: 13·3-25·5). In the rabies vaccine arm, anti-CS seropositivity and anti-CS GMT remained low throughout the study period (table 4 and supplement figure 3, page 2).

At baseline, seroprotective titres of anti-Hepatitis B (anti-HBs) was similar between the RTS,S/AS01 arm at $57.1 \%$ (95\%CI: 45.4-68.4) and the rabies vaccine arm at 54.8\% (95\%CI: 42.7-66.5). The anti-HBs GMTs were $24.1 \mathrm{mIU} / \mathrm{ml}$ in the RTS,S/AS01 arm and $19.2 \mathrm{mIU} / \mathrm{ml}$ in the rabies vaccine arm. One month post-dose 3,100\% (95\%CI: 95.1-100) of subjects in the RTS,S/AS01 arm and 52.3\% (95\%CI: 39.5-64.9) in the rabies vaccine arm were seroprotected for anti-HBs. The anti-HBs GMTs were $13637.6 \mathrm{mIU} / \mathrm{ml}$ in the RTS,S/AS01 arm and $19.9 \mathrm{mIU} / \mathrm{ml}$ in the rabies vaccine arm. Twelve months post-dose 3,100\% (95\%CI: 94.9-100) of subjects in the RTS,S/AS01 arm and 39.1\% (95\%CI: 27.1-52.1) in the rabies vaccine arm were seroprotected for anti-HBs. The anti-HBs GMTs were $2294.8 \mathrm{mIU} / \mathrm{ml}$ and $11.8 \mathrm{mIU} / \mathrm{ml}$ in the RTS,S/AS01 arm and the rabies vaccine arm, respectively (supplement table 9, page 11).

\section{Efficacy and other study endpoints}

Children in the RTS,S/AS01 arm experienced $0 \cdot 541$ cases of clinical malaria per person year, compared with 0.886 cases per person year in the rabies vaccine arm, giving a VE estimate of $37 \cdot 2 \%$ (95\% CI: 26.5-68.8) (supplement tables 10 and 11, pages 12 and 13). VE was consistent across different malaria case definitions (supplement table 4, page 5). Severe malaria occurred in 1/87 (1\%) of children in the RTS,S/AS01 arm and 8/93 (8\%) of children in the rabies vaccine arm. Malaria hospitalization in the 
RTS,S/AS01 arm occurred in 2/87 (2\%) compared with 8/93 (8\%) of children in the rabies vaccine arm (supplement table 12, page 14). No effect was seen against prevalent parasitaemia or anaemia but more cases of anaemia were reported in the rabies vaccine arm over 14 months (table 2). Growth as measured by height, weight and MUAC was comparable between vaccine arms (supplement table 13, page 14). 


\section{DISCUSSION}

Recent data have shown the RTS,S/AS01 vaccine to be well tolerated and moderately efficacious in preventing malaria in children living in a range of malaria transmission settings across sub-Saharan Africa. $^{4-6,21}$ We now show that RTS, S/AS01 malaria vaccine is well tolerated and is immunogenic in HIV-infected children.

In the RTS, S/AS01 arm, one related SAE, a febrile convulsion occurred within seven days of vaccination. The child fully recovered and this event was consistent with findings from the large multisite, phase III trial. ${ }^{6}$ SAEs were reported more frequently in this HIV infected population than in the large multi-site, phase 3 trial, both for children and infants. In children in the large multi-site trial, $17.6 \%$ of RTS,S/AS01 recipients and $21.6 \%$ of rabies vaccine recipients had at least one SAE associated with the primary vaccination series. ${ }^{5,6}$ This is not surprising given that HIV infected children are at higher risk of common childhood illnesses than non-HIV infected children. The imbalance in meningitis reported in the larger multi-site, phase III trial, ${ }^{6}$ was not seen in this comparatively small trial. Although not statistically significant, a higher proportion of RTS, S/AS01 vaccine recipients experienced at least one SAE during the initial 30 days following vaccination when compared with rabies vaccine recipients; mainly due to pneumonia, febrile convulsions and Salmonella sepsis, with cases balanced between vaccination arms by study end. Exploratory analysis of pneumonia cases showed no clustering in time to onset with any dose and no clear pattern emerged to suggest a true relationship between pneumonia and RTS,S/AS01 vaccination. The increase in pneumonia cases following vaccination may be a chance finding, but could be explored further in the RTS,S/AS01 implementation pilots or phase IV studies. The RTS,S/AS01 vaccine was more reactogenic than the rabies vaccine although few grade 3 reactions in either study arms.

RTS,S/AS01 vaccination was not associated with HIV disease progression in terms of CD4+ T-cell, HIV VL and/or WHO HIV clinical classification. Overall, there was a trend for decreasing HIV VL over time but with a higher median HIV VL among children enrolled in the RTS,S/AS01 arm compared to the rabies vaccine arm. The largest difference in VL was reported at 6 months post-dose 3. While a possible association between delayed HIV VL clearance or a transient increase in HIV VL and RTS,S/AS01 vaccine which has a hepatitis B component ${ }^{9,10}$ could not be ruled out, exploratory statistical modelling suggested that the differences in change from baseline HIV VL were not associated with vaccine assignment but was related to baseline HIV VL, site, early CTX use and ART use. Transient increases in HIV RNA plasma levels have been described following immunization with several different vaccines such as pneumococcal ${ }^{22}$, influenza ${ }^{23}$ and hepatitis B vaccines. ${ }^{24}$ These transient, clinically non-significant increases in viral load are counterbalanced by the benefit of vaccination and therefore have not resulted in any contraindications to vaccination. ${ }^{25}$ 
Studies have demonstrated that immune responses to vaccines such as hepatitis B virus and influenza are reduced in patients with HIV infection. ${ }^{26,27}$ In this trial, the anti-CS antibody titers at baseline were low in both study arms and remained low in the rabies vaccine arm throughout the trial. One month post-dose 3, $100 \%$ of children who received the RTS,S/AS01 vaccine were positive for anti-CS antibodies, with a GMT level of 329.2 EU/mL. Twelve months post-dose 3, 99\% of children in the RTS,S/AS01 arm remained seropositive with anti-CS GMT at $18.4 \mathrm{EU} / \mathrm{mL}$. This seroconversion pattern is similar to observations in the larger RTS,S/AS01 multi-site, phase III vaccine trial. ${ }^{5,6}$ However, anti-CS GMT is lower than in the larger multi-site, phase III trial, where the GMT after vaccine dose 3 was $621 \mathrm{EU} / \mathrm{mL}$ in children first vaccinated at age 5-17 months. ${ }^{6}$ In this trial, $80 \%$ of enrolled children were aged 5-17 months and therefore the anti-CS GMT values were most comparable to that age category in the larger multi-site, phase III trial. ${ }^{6}$ The RTS,S/AS01 vaccine was immunogenic in this trial but had lower anti-CS GMT at one month post-dose 3 compared to observations in children aged 5-17 months at the same study sites in the large multi-site, phase III trial. ${ }^{6}$ The anti-CS GMT at one month post-dose 3 was 745.1 EU/mL at the KEMRI/WRAIR site and 708.6 EU/mL at the KEMRI/CDC site. ${ }^{28}$ The lower anti-CS GMT in this trial compared with the RTS,S/AS01 multi-site, phase III trial is likely attributable to a reduced immunological response due to HIV infection, a phenomenon described with other vaccines. ${ }^{13}$

Although this study captured all malaria cases prospectively, it was not primarily powered to measure vaccine efficacy against clinical or severe malaria. Although statistically non-significant, children who received the RTS,S/AS01 vaccine experienced fewer episodes of clinical malaria, severe malaria, malaria hospitalizations and anaemia than did children who received rabies vaccine. These findings were observed despite high uptake of malaria preventive measures, specifically insecticide-treated nets (ITN) and daily cotrimoxazole for the prevention of opportunistic infections, which has also been shown to protect against malaria. ${ }^{29}$ These observations which need to be interpreted with caution given the wide confidence intervals and small sample size, suggest that RTS,S/AS01 might provide some protection against malaria in HIV infected individuals on ART and daily cotrimoxazole, but this would need to be confirmed through additional studies . The lower immunogenicity seen in HIV-infected children in this trial when compared with that seen in similar aged children in the multi-site phase III trial, and the evidence for a statistical correlation between anti-CS antibody responses and protection seen in most studies $^{30}$, indicates that vaccine-induced protection may be lower in HIV-infected children than in HIV negative children. 
Our trial had several limitations that affect its generalizability to all HIV infected children. Most children, $73 \%$, were on ART at the time of screening, increasing to $97 \%$ shortly after initial vaccination, and all children were on ART by 6 months post-dose 3. Therefore, these data describe the safety profile and immunogenicity of RTS,S/AS01 vaccine when given to HIV infected children who are on ART, either at first vaccination, or who initiate ART soon thereafter. In terms of immunogenicity assessment, the most relevant assay according to extensive past evaluation has been used in this study ${ }^{19}$, but we did not study functionality, cell-mediated immunity or other immune factors which may differ significantly between HIV positive and negative individuals. Adverse events associated with RTS,S/AS01 vaccine administration to HIV infected children not on ART cannot be excluded. Our trial excluded HIV infected children with WHO HIV clinical classification of 3 or 4; thus RTS,S/AS01 vaccine safety in children with advanced HIV disease is unknown. In children with elevated HIV VL, antiretroviral resistance testing was not carried out as this is not routinely done in resource limited settings but this is unlikely to have affected our evaluation of vaccine safety and immunogenicity. Children $<5$ months were underrepresented in our trial but this should not be a problem when considering the WHO recommended pilot implementation studies in the age category 5-17 months. The trial had a relatively small sample size and was conducted in western Kenya in a homogenous population largely from a single ethnic group living in an area of high malaria transmission. ${ }^{14}$ We therefore cannot exclude the possibility that results might differ in other populations. The WHO recommended pilot implementation studies ${ }^{8}$ or other future studies would provide a platform to further monitor the safety and efficacy of RTS,S/AS01 in populations in other settings.

In conclusion, this trial demonstrated that RTS,S/AS01 vaccine was not associated with clinically significant safety problems and was immunogenic when administered to children with WHO clinical stage 1 or 2 HIV disease in the context of high ART and CTX use. These findings demonstrate that children with WHO clinical stage 1 or 2 HIV disease could be included in future RTS,S/AS01 vaccination programs, should the vaccine be recommended for wide-scale use.

\section{Contributors}

BA, NA,MH, KI, SK, AL, DLa, DLe, ML ,CO, BO, MOn, AO, KO, LO, WO, NO, EO, JO, LS, JV designed the study; JA,BA, NA, JC, YGM, MH, SK, CO, BO, MOn, AO, KO, LO, WO, NO, EO, JO, MOz, LS, EAU collected the data; BA, YGM, MH, DH, AL, ML, MOn, LO, WO, EAU, JV performed or supervised the analysis; NA, YGM ,MH, DH, SK, AL, DLa, DLe, ML, CO, BO, MOn, LO, WO, NO, MOz, LS, EAU, JV interpreted the data; YGM, MH,DH, AL, DLe, MOn, LO and NO were the core writing team for the manuscript. All authors reviewed and commented on a draft version of the manuscript and gave final approval for it to be submitted for publication. 


\section{Declaration of interests}

The trial was sponsored by GlaxoSmithKline Biologicals SA, the vaccine developer and manufacturer, and funded by both GlaxoSmithKline Biologicals SA and the PATH Malaria Vaccine Initiative (MVI). All centers declare receiving a grant from MVI for running the trial. Author travel and accommodation related to this trial were financed by both GSK and MVI. GlaxoSmithKline Biologicals SA received a grant from MVI to run the trial. MVI received a grant from the Bill and Melinda Gates Foundation to run this trial and to compensate MVI authors for trial-related travel.

YGM, DLa, AL, ML, JV are employees, and DH a former consultant of the GSK group of companies. EAU is a former WHO TDR fellow at GSK. YGM, DLa, AL, ML, JV have shares/stock options in the GSK group of companies. KI, CO are employees, and DLe a former employee of the PATH Malaria Vaccine Initiative. LO declares that his institution received grant from GSK and MVI to conduct other malaria studies. LO declares that he received support from GSK to attend scientific conferences and funding from GSK for his Trust in Science Africa grant. NO declares that she received support from GSK to attend scientific conferences.

\section{Acknowledgments}

The trial was sponsored by GlaxoSmithKline Biologicals SA and was funded by both GlaxoSmithKline Biologicals SA and the PATH MVI. The MVI received a grant from the Bill \& Melinda Gates Foundation to run this trial and to compensate MVI authors for trial-related travel. GlaxoSmithKline Biologicals SA developed and manufactured the vaccine. The opinions and assertions herein are the views of authors and not those of KEMRI, the Department of Defense, Centers for Disease Control and Prevention or US Government.

The authors thank the following:

The children and their families and communities who generously participated in this trial, the study team members at each site, staff of the health facilities in the study areas and the Jaramogi Oginga Odinga Teaching and Referral Hospital, Kisumu, Kenya, and the national and local government authorities for their guidance and support for the implementation of the trial.

Those listed below by study center who contributed in various ways to the trial:

KEMRI/CDC Research and Public Health Collaboration, Kisumu, Kenya: Jael Asewe, Grace Chumbe, Patrick Kachur, Vincent Muturi-Kioi, Christina Obiero, Brian Obunga, Cecilia Ochieng, John Vulule, the Kenyan Ministry of Health, the MOH staff at Siaya District Hospital, Ting Wang'i and Kogelo Health 
Centers, Ngiya Mission Hospital, the Siaya District Health Management Team for their support during the trial, and the children and parents in Siaya who participated in this important trial.

KEMRI - Walter Reed Project, Kisumu, Kenya: Barrack Agutu, Consolata Appida, Carolyne Laboso, Irène Miruka, Jacob Nyariro, Dorothy Odera, George Odongo, Mary Omondi, Caroline Ongoro, Agnès Onyango, Lilian Otieno, Victorine Owira, Ruth Wasuna

GSK Vaccines, Belgium: Xavier Druart, Elodie Garric, Ioana Cristina Ilea, Sarah Liégeois, Thomas Moens, Myriam Wilbaux (XPE Pharma and Science on behalf of GSK Vaccines) for editorial support and publication coordination. 


\section{Panel: Research in context}

\section{Evidence before this study}

The RTS,S/AS01 malaria vaccine may become the first malaria vaccine licensed for use in African children. Because of the considerable geographical overlap between malaria and HIV infection, it is important to understand whether the RTS,S/AS01 vaccine is safe and immunogenic when administered to HIV infected children. We searched for randomized controlled trials of malaria vaccine candidates in HIV infected populations on PubMed, the Cochrane Library and other relevant data sources for the period 01 January 1984 to 31 August 2015. PubMed was searched using the MeSH terms: (("malaria vaccines"[MeSH Terms] OR ("malaria"[All Fields] AND "vaccines"[All Fields]) OR "malaria vaccines"[All Fields] OR ("malaria"[All Fields] AND "vaccine"[All Fields]) OR "malaria vaccine"[All Fields]) AND (rts[All Fields] AND s[All Fields])) AND ("hiv"[MeSH Terms] OR "hiv"[All Fields]). For the Cochrane Library and other data sources, the key search terms used were "RTS, S", "Malaria Vaccines", "HIV"AND "Clinical Trials". The 7 papers identified did not contain clinical data of administration of a malaria vaccine candidate in a confirmed HIV positive population. Even though the large multi-site phase 3 RTS,S/AS01 trial enrolled some HIV positive children, to our knowledge, this is the first trial to specifically report safety and immunogenicity results of a malaria vaccine in HIV infected population.

\section{Added value of this study}

The RTS,S/AS01 malaria vaccine has been evaluated for safety, immunogenicity and efficacy in multiple paediatric trials over several years. This study for the first time provides additional important information on the safety and immunogenicity of RTS,S/AS01 in a population of HIV infected infants and children.

\section{Implications of all available evidence}

The results of this safety and immunogenicity trial demonstrate that HIV-infected children should not be excluded from potential future vaccination with RTS,S/AS01. These results will contribute to the body of evidence required for policy formulation and regulatory decisions regarding the RTS,S/AS01 malaria vaccine. 


\section{REFERENCES}

1. World Malaria Report 2015. WHO 2015.

2. Greenwood BM, Fidock DA, Kyle DE, Kappe SH, Alonso PL, et al. Malaria: Progress, perils and prospects for eradication. J Clin Invest 118: 1266, 2008.

3. UNAIDS Global Report 2013.

4. The RTS, S Clinical Trials Partnership. Efficacy and safety of RTS,S/AS01 malaria vaccine with or without a booster dose in infants and children in Africa: final results of a phase 3, individually randomised, controlled trial. Lancet 2015; 386: 31-45.

5. The RTS, S Clinical Trials Partnership. A phase 3 trial of RTS, S/AS01 malaria vaccine in African infants. N Engl J Med 2012; 367: 2284-95.

6. The RTS, S Clinical Trials Partnership. First results of phase 3 trial of RTS, S/AS01 malaria vaccine in African children. N Engl J Med 2011; 365: 1863-75.

7. First malaria vaccine receives positive scientific opinion from EMA. http://www.ema.europa.eu/docs/en_GB/document_library/Press_release/2015/07/WC500190447.pdf. Accessed February 2016.

8. Malaria Vaccine: WHO position paper-January 2016. Weekly Epidemiological Record. No. 4, 2016, 91, 33-52.

9. Cohen J, Nussenzweig V, Nussenzweig R, Vekemans J and Leach A. From the circumsporozoite protein to the RTS, S/AS candidate vaccine. Human Vaccines 6:1, 90-96; January 2010.

10. Casaresa S, Brumeanub TD, and Richie TL. The RTS, S malaria vaccine. Vaccine 28 (2010) 48804894.

11. Malaria and HIV interactions and their implications for public health policy. Report of a Technical Consultation, World Health Organization, Geneva, Switzerland, 2004.

12. Cuadros DF, Branscum AJ, Crowley PH. HIV-malaria co-infection: effects of malaria on the prevalence of HIV in East sub-Saharan Africa. Int J Epidemiol 2011; 40: 931-9.

13. Moss WJ, et al. Immunization of children at risk of infection with human immunodeficiency virus. Bulletin of the World Health Organization 2003, 81 (1).

14. Kenya Annual Malaria Report July 2012-June 2013, Ministry of Health.

15. Kohler PK, Okanda J, Kinuthia J, et al. (2014) Community-Based Evaluation of PMTCT Uptake in Nyanza Province, Kenya. PLoS ONE 9(10):e110110. doi:10.1371/journal.pone.0110110. .

16. Thomas TK, Masaba R, Borkowf CB, et al. (2011) Triple-Antiretroviral Prophylaxis to Prevent Mother-To-Child HIV Transmission through Breastfeeding-The Kisumu Breastfeeding Study, Kenya: A Clinical Trial. PLoS Med 8(3): e1001015. doi:10.1371/journal.pmed.1001015. 
17. Bonhoeffer J, Menkes J, Gold MS, et al. Generalized convulsive seizure as an adverse event following immunization: case definition and guidelines for data collection, analysis, and presentation. Vaccine 2004; 22: 557-62.

18. Verbal autopsy standards: ascertaining and attributing cause of death. World Health Organization, Geneva, Switzerland, 2007.

19. Clement F, Dewar V, Van Braeckel E, et al. Validation of an enzyme-linked immunosorbent assay for the quantification of human IgG directed against the repeat region of the circumsporozoite protein of the parasite Plasmodium falciparum. Malar J. 2012 Nov 22; 11:384. doi: 10.1186/1475-2875-11-384.

20. Vickers AJ. The use of percentage change from baseline as an outcome in a controlled trial is statistically inefficient: a simulation study. BMC Med Res Methodol 2001, 1: 6.

21. Vekemans J, Guerra Y, Lievens M, et al. Pooled analysis of safety data from pediatric Phase II RTS, S/AS malaria candidate vaccine trials. Hum Vaccin 2011; 7: 1309-16.

22. Brichacek B, Swindells S, Janoff EN, Pirruccello S, Stevenson M. Increased plasma human immunodeficiency virus type 1 burden following antigenic challenge with pneumococcal vaccine. $J$ Infect Dis 1996; 174: 1191-9.

23. Gunthard HF, Wong JK, Spina CA, et al. Effect of Influenza Vaccination on Viral Replication and Immune Response in Persons Infected with Human Immunodeficiency Virus Receiving Potent Antiretroviral Therapy. The Journal of Infectious Diseases 2000; 181:522-31.

24. Cheeseman SH, Davaro RE, Ellision, RT 3rd. Hepatitis B vaccination and plasma HIV-1 RNA. $N$ Engl JMed 1996; 334: 1272.

25. British HIV Association Guidelines on the use of Vaccines in HIV-Positive Adults 2015.

26. Kim HN, Harrington RD, Crane HM, Dhanireddy S, Dellit TH, Spach DH. Hepatitis B vaccination in HIV-infected adults: current evidence, recommendations and practical considerations. Int J STD AIDS 2009; 20: 595-600.

27. Kroon FP, van Dissel JT, de Jong JC, Zwinderman K, van Furth R. Antibody response after influenza vaccination in HIV-infected individuals: a consecutive 3-year study. Vaccine 2000; 18: 3040-9.

28. The RTS,S Clinical Trials Partnership (2014). Efficacy and Safety of the RTS,S/AS01 Malaria Vaccine during 18 Months after Vaccination: A Phase 3 Randomized, Controlled Trial in Children and Young Infants at 11 African Sites. PLoS Med 11(7): e1001685.

doi:10.1371/journal.pmed.1001685.

29. Manyando C, Njunju EM, D’Alessandro U, Van Geertruyden JP. Safety and efficacy of cotrimoxazole for treatment and prevention of Plasmodium falciparum malaria: a systematic review. PLoS One 2013, 8: e56916. 
30. Ockenhouse CF, Regules J, Tosh D, Cowden J, et al. Ad35.CS.01-RTS,S/AS01 Heterologous Prime Boost Vaccine Efficacy against Sporozoite Challenge in Healthy Malaria-Naïve Adults. PLoS One. 2015 Jul 6; 10 (7):e0131571. doi: 10.1371/journal.pone.0131571. eCollection 2015. 


\section{TABLES}

Table 1: Baseline characteristics of children at screening (intention-to-treat population)

\begin{tabular}{|c|c|c|c|}
\hline Characteristic & Category & $\begin{array}{c}\text { RTS,S/AS01 vaccine } \\
\qquad \begin{array}{c}\text { N=99 }\end{array}\end{array}$ & $\begin{array}{l}\text { Rabies vaccine } \\
\qquad \mathbf{N}=101\end{array}$ \\
\hline & 6 weeks to 4 months & $20(20 \cdot 2)$ & $20(19 \cdot 8)$ \\
\hline Age category, $\mathrm{n}(\%)$ & 5 to 17 months & $79(79 \cdot 8)$ & $81(80 \cdot 2)$ \\
\hline Gender, n (\%) & Male & $42(42 \cdot 4)$ & $56(55 \cdot 4)$ \\
\hline Height-for-age z-score, mean \pm SD & & $-1 \cdot 67 \pm 1 \cdot 20$ & $-1.98 \pm 1 \cdot 37$ \\
\hline Weight-for-age z-score, mean \pm SD & & $-1 \cdot 38 \pm 1 \cdot 13$ & $-1 \cdot 67 \pm 1 \cdot 19$ \\
\hline MUAC z-score, mean \pm SD & & $-0 \cdot 65 \pm 1 \cdot 13$ & $-0 \cdot 90 \pm 1 \cdot 19$ \\
\hline Haemoglobin $[\mathrm{g} / \mathrm{dL}]$, mean $\pm \mathrm{SD}$ & & $9 \cdot 74 \pm 1 \cdot 31$ & $9 \cdot 60 \pm 1 \cdot 14$ \\
\hline Moderate anaemia, n (\%) & $\geq 5$ to $<8 \mathrm{~g} / \mathrm{dL}$ & $5(5.1)$ & $5(5.0)$ \\
\hline Bed net use*, n (\%) & & $86(98 \cdot 9)$ & $87(96 \cdot 7)$ \\
\hline ART treatment status dose $1(\%)$ & & $73(73.7)$ & $73(72.3)$ \\
\hline Cotrimoxazole treatment status dose $1(\%)$ & & $92(92.9)$ & $92(91.1)$ \\
\hline KEMRI/WRAIR, Kisumu & & 59 & 64 \\
\hline KEMRI/CDC, Siaya & & 40 & 37 \\
\hline
\end{tabular}

$\mathrm{N}=$ number of children. $\mathrm{n}(\%)=$ number (percentage) of children in a given category. $\mathrm{SD}=$ standard deviation. MUAC=middle upper arm circumference. $*$ Bed net use reported at month 14. 
Table 2: Serious adverse events during 14 months after the first vaccine dose in children 6 weeks to 17 months at enrolment (intention-to-treat population)

\begin{tabular}{|c|c|c|c|c|}
\hline \multirow{2}{*}{ Serious adverse event } & \multicolumn{2}{|c|}{$\begin{array}{c}\begin{array}{c}\text { RTS,S/AS01 vaccine } \\
\text { N=99 }\end{array}\end{array}$} & \multicolumn{2}{|c|}{$\begin{array}{c}\text { Rabies vaccine } \\
\quad \mathrm{N}=101\end{array}$} \\
\hline & $\begin{array}{l}\text { no. of } \\
\text { children }\end{array}$ & $\%(95 \% \mathrm{CI})$ & $\begin{array}{l}\text { no. of } \\
\text { children }\end{array}$ & $\%(95 \% \mathrm{CI})$ \\
\hline \multicolumn{5}{|l|}{ All children } \\
\hline At least one SAE & 41 & $41 \cdot 4(31 \cdot 6-51 \cdot 8)$ & 37 & $36 \cdot 6(27 \cdot 3-46 \cdot 8)$ \\
\hline Fatal SAE & 5 & $5 \cdot 1(1 \cdot 7-11 \cdot 4)$ & 4 & $4 \cdot 0(1 \cdot 1-9 \cdot 8)$ \\
\hline At least one SAE within 30 days after vaccination & 20 & $20 \cdot 2(12 \cdot 8-29 \cdot 5)$ & 12 & $11 \cdot 9(6 \cdot 3-19 \cdot 8)$ \\
\hline \multicolumn{5}{|l|}{ Events reported in all children* } \\
\hline \multicolumn{5}{|l|}{ Blood and lymphatic system disorders } \\
\hline Anaemia & 1 & $1 \cdot 0(0 \cdot 0-5 \cdot 5)$ & 7 & $6 \cdot 9(2 \cdot 8-13 \cdot 8)$ \\
\hline \multicolumn{5}{|l|}{ Gastrointestinal disorders } \\
\hline Enteritis & 0 & $0 \cdot 0(0 \cdot 0-3 \cdot 7)$ & 1 & $1 \cdot 0(0 \cdot 0-5 \cdot 4)$ \\
\hline \multicolumn{5}{|l|}{$\underline{\text { General disorders and administration site conditions }}$} \\
\hline Pyrexia & 1 & $1 \cdot 0(0 \cdot 0-5 \cdot 5)$ & 0 & $0 \cdot 0(0 \cdot 0-3 \cdot 6)$ \\
\hline \multicolumn{5}{|l|}{$\underline{\text { Hepatobiliary disorders }}$} \\
\hline Hepatitis & 1 & $1 \cdot 0(0 \cdot 0-5 \cdot 5)$ & 0 & $0 \cdot 0(0 \cdot 0-3 \cdot 6)$ \\
\hline \multicolumn{5}{|l|}{$\underline{\text { Infections and infestations }}$} \\
\hline Abscess & 1 & $1 \cdot 0(0 \cdot 0-5 \cdot 5)$ & 0 & $0 \cdot 0(0 \cdot 0-3 \cdot 6)$ \\
\hline Amoebiasis & 0 & $0 \cdot 0(0 \cdot 0-3 \cdot 7)$ & 1 & $1 \cdot 0(0 \cdot 0-5 \cdot 4)$ \\
\hline Bronchiolitis & 0 & $0 \cdot 0(0 \cdot 0-3 \cdot 7)$ & 2 & $2 \cdot 0(0 \cdot 2-7 \cdot 0)$ \\
\hline Cellulitis & 0 & $0 \cdot 0(0 \cdot 0-3 \cdot 7)$ & 1 & $1 \cdot 0(0 \cdot 0-5 \cdot 4)$ \\
\hline Gastroenteritis & 21 & $21 \cdot 2(13 \cdot 6-30 \cdot 6)$ & 19 & $18 \cdot 8(11 \cdot 7-27 \cdot 8)$ \\
\hline Gastroenteritis salmonella & 0 & $0 \cdot 0(0 \cdot 0-3 \cdot 7)$ & 1 & $1 \cdot 0(0 \cdot 0-5 \cdot 4)$ \\
\hline Helminthic infection & 2 & $2 \cdot 0(0 \cdot 2-7 \cdot 1)$ & 0 & $0 \cdot 0(0 \cdot 0-3 \cdot 6)$ \\
\hline Malaria & 5 & $5 \cdot 1(1 \cdot 7-11 \cdot 4)$ & 10 & $9 \cdot 9(4 \cdot 9-17 \cdot 5)$ \\
\hline Measles & 0 & $0 \cdot 0(0 \cdot 0-3 \cdot 7)$ & 3 & $3 \cdot 0(0 \cdot 6-8 \cdot 4)$ \\
\hline Meningitis Haemophilus & 0 & $0 \cdot 0(0 \cdot 0-3 \cdot 7)$ & 1 & $1 \cdot 0(0 \cdot 0-5 \cdot 4)$ \\
\hline Mycobacterium avium complex infection & 0 & $0 \cdot 0(0 \cdot 0-3 \cdot 7)$ & 1 & $1 \cdot 0(0 \cdot 0-5 \cdot 4)$ \\
\hline
\end{tabular}




\begin{tabular}{|c|c|c|c|c|}
\hline Oral candidiasis & 5 & $5 \cdot 1(1 \cdot 7-11 \cdot 4)$ & 5 & $5 \cdot 0(1 \cdot 6-11 \cdot 2)$ \\
\hline Oropharyngeal candidiasis & 0 & $0 \cdot 0(0 \cdot 0-3 \cdot 7)$ & 1 & $1 \cdot 0(0 \cdot 0-5 \cdot 4)$ \\
\hline Otitis media & 1 & $1 \cdot 0(0 \cdot 0-5 \cdot 5)$ & 4 & $4 \cdot 0(1 \cdot 1-9 \cdot 8)$ \\
\hline Pneumococcal sepsis & 0 & $0 \cdot 0(0 \cdot 0-3 \cdot 7)$ & 3 & $3 \cdot 0(0 \cdot 6-8 \cdot 4)$ \\
\hline Pneumocystis jiroveci pneumonia & 1 & $1 \cdot 0(0 \cdot 0-5 \cdot 5)$ & 0 & $0 \cdot 0(0 \cdot 0-3 \cdot 6)$ \\
\hline Pneumonia & 23 & $23 \cdot 2(15 \cdot 3-32 \cdot 8)$ & 23 & $22 \cdot 8(15 \cdot 0-32 \cdot 2)$ \\
\hline Pulmonary tuberculosis & 2 & $2 \cdot 0(0 \cdot 2-7 \cdot 1)$ & 2 & $2 \cdot 0(0 \cdot 2-7 \cdot 0)$ \\
\hline Salmonella sepsis & 7 & $7 \cdot 1(2 \cdot 9-14 \cdot 0)$ & 6 & $5 \cdot 9(2 \cdot 2-12 \cdot 5)$ \\
\hline Sepsis & 0 & $0 \cdot 0(0 \cdot 0-3 \cdot 7)$ & 1 & $1 \cdot 0(0 \cdot 0-5 \cdot 4)$ \\
\hline Tuberculosis & 2 & $2 \cdot 0(0 \cdot 2-7 \cdot 1)$ & 0 & $0 \cdot 0(0 \cdot 0-3 \cdot 6)$ \\
\hline Upper respiratory tract infection & 0 & $0 \cdot 0(0 \cdot 0-3 \cdot 7)$ & 1 & $1 \cdot 0(0 \cdot 0-5 \cdot 4)$ \\
\hline Urinary tract infection & 2 & $2 \cdot 0(0 \cdot 2-7 \cdot 1)$ & 2 & $2 \cdot 0(0 \cdot 2-7 \cdot 0)$ \\
\hline Varicella & 1 & $1 \cdot 0(0 \cdot 0-5 \cdot 5)$ & 0 & $0 \cdot 0(0 \cdot 0-3 \cdot 6)$ \\
\hline Viral infection & 0 & $0 \cdot 0(0 \cdot 0-3 \cdot 7)$ & 1 & $1 \cdot 0(0 \cdot 0-5 \cdot 4)$ \\
\hline \multicolumn{5}{|l|}{ Metabolism and nutrition disorders } \\
\hline Dehydration & 0 & $0 \cdot 0(0 \cdot 0-3 \cdot 7)$ & 2 & $2 \cdot 0(0 \cdot 2-7 \cdot 0)$ \\
\hline Hypokalaemia & 0 & $0 \cdot 0(0 \cdot 0-3 \cdot 7)$ & 1 & $1 \cdot 0(0 \cdot 0-5 \cdot 4)$ \\
\hline Kwashiorkor & 1 & $1 \cdot 0(0 \cdot 0-5 \cdot 5)$ & 0 & $0 \cdot 0(0 \cdot 0-3 \cdot 6)$ \\
\hline Malnutrition & 7 & $7 \cdot 1(2 \cdot 9-14 \cdot 0)$ & 5 & $5 \cdot 0(1 \cdot 6-11 \cdot 2)$ \\
\hline \multicolumn{5}{|l|}{ Nervous system disorders } \\
\hline Convulsion & 2 & $2 \cdot 0(0 \cdot 2-7 \cdot 1)$ & 0 & $0 \cdot 0(0 \cdot 0-3 \cdot 6)$ \\
\hline Febrile convulsion & 10 & $10 \cdot 1(5 \cdot 0-17 \cdot 8)$ & 13 & $12 \cdot 9(7 \cdot 0-21 \cdot 0)$ \\
\hline \multicolumn{5}{|c|}{ Respiratory, thoracic and mediastinal disorders } \\
\hline Pneumonia aspiration & 1 & $1 \cdot 0(0 \cdot 0-5 \cdot 5)$ & 1 & $1 \cdot 0(0 \cdot 0-5 \cdot 4)$ \\
\hline
\end{tabular}

$\mathrm{SAE}=$ serious adverse event. $\mathrm{N}=$ number of children with at least one administered dose. $\mathrm{CI}=$ confidence interval.

*Events are listed according to the preferred terms in the Medical Dictionary for Regulatory Activities. 
Table 3: HIV disease progression (intention-to-treat population)

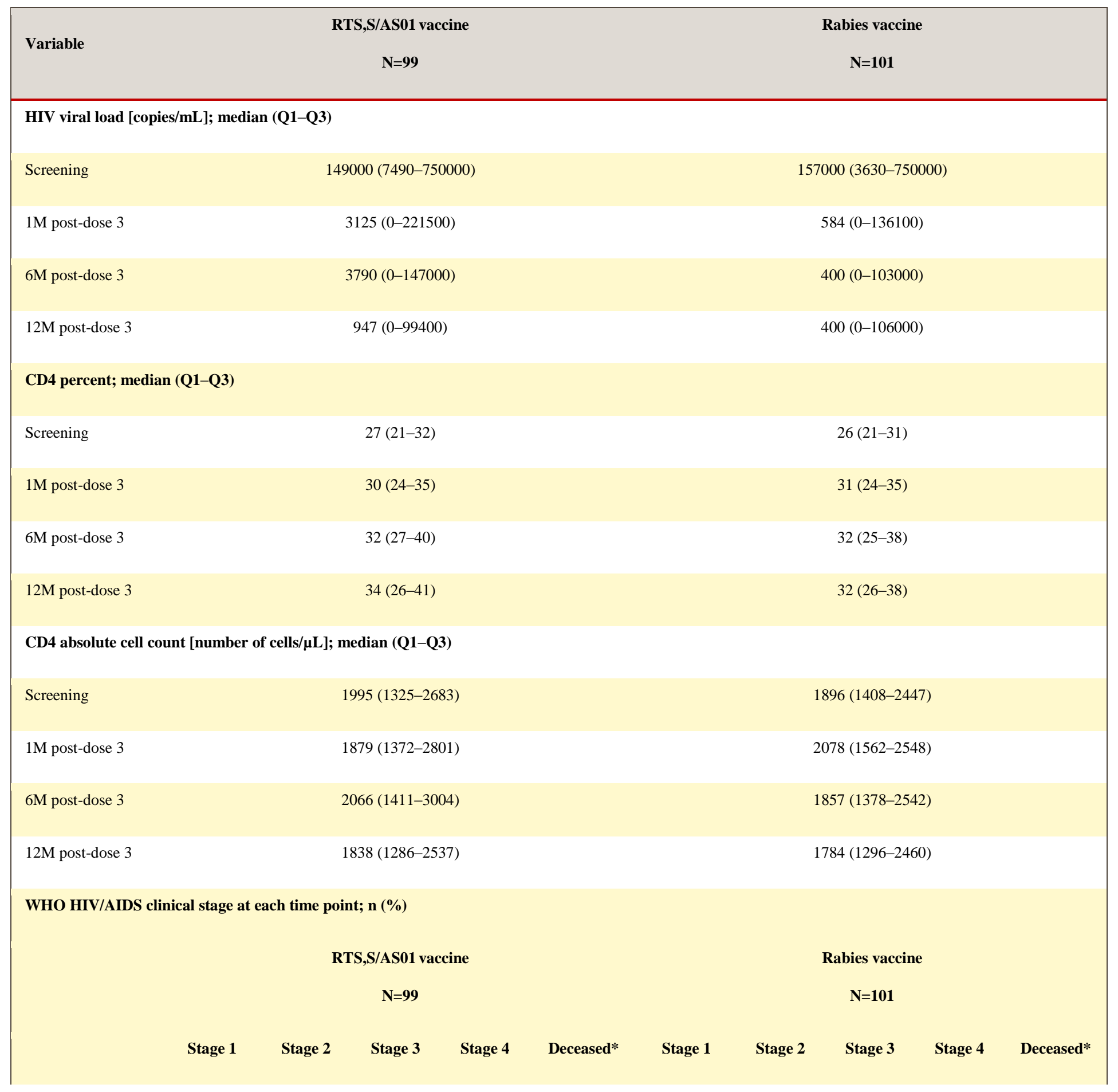




\begin{tabular}{|lcccccccccc} 
Screening & $81(81 \cdot 8)$ & $18(18 \cdot 2)$ & $0(0 \cdot 0)$ & $0(0 \cdot 0)$ & $0(0 \cdot 0)$ & $82(81 \cdot 2)$ & $19(18 \cdot 8)$ & $0(0 \cdot 0)$ & $0(0 \cdot 0)$ & $0(0 \cdot 0)$ \\
$1 \mathrm{M}$ post-dose 1 & $75(76 \cdot 5)$ & $19(19 \cdot 4)$ & $2(2 \cdot 0)$ & $0(0 \cdot 0)$ & $2(2 \cdot 0)$ & $72(71 \cdot 3)$ & $27(26 \cdot 7)$ & $1(1 \cdot 0)$ & $0(0 \cdot 0)$ & $1(1 \cdot 0)$ \\
$1 \mathrm{M}$ post-dose 2 & $79(81 \cdot 4)$ & $15(15 \cdot 5)$ & $1(1 \cdot 0)$ & $0(0 \cdot 0)$ & $2(2 \cdot 1)$ & $77(77 \cdot 0)$ & $20(20 \cdot 0)$ & $2(2 \cdot 0)$ & $0(0 \cdot 0)$ & $1(1 \cdot 0)$ \\
$1 \mathrm{M}$ post-dose 3 & $76(80 \cdot 9)$ & $12(12 \cdot 8)$ & $4(4 \cdot 3)$ & $0(0 \cdot 0)$ & $2(2 \cdot 1)$ & $76(78 \cdot 4)$ & $19(19 \cdot 6)$ & $0(0 \cdot 0)$ & $0(0 \cdot 0)$ & $2(2 \cdot 1)$ \\
$6 \mathrm{M}$ post-dose 3 & $74(82 \cdot 2)$ & $12(13 \cdot 3)$ & $0(0 \cdot 0)$ & $0(0 \cdot 0)$ & $4(4 \cdot 4)$ & $72(76 \cdot 6)$ & $16(17 \cdot 0)$ & $3(3 \cdot 2)$ & $0(0 \cdot 0)$ & $3(3 \cdot 2)$ \\
$12 \mathrm{M}$ post-dose 3 & $74(80 \cdot 4)$ & $10(10 \cdot 9)$ & $3(3 \cdot 3)$ & $0(0 \cdot 0)$ & $5(5 \cdot 4)$ & $69(73 \cdot 4)$ & $18(19 \cdot 1)$ & $1(1 \cdot 1)$ & $2(2 \cdot 1)$ & $4(4 \cdot 3)$
\end{tabular}

*The number of deceased children at each time point is cumulative and the overall mortality is presented in Table 2 as the number of children with at least one fatal serious adverse event. HIV=human immunodeficiency virus. Q1 and Q3 represent interquartile ranges. $\mathrm{N}=$ number of children. $\mathrm{M}=$ month(s). WHO=World Health Organization. AIDS=acquired immunodeficiency syndrome. $\mathrm{n}$ $(\%)=$ number (percentage) of children in a given category.

Table 4: Seropositivity rates and geometric mean titres for anti-circumsporozoite antibodies (ATP population for immunogenicity)

\begin{tabular}{|c|c|c|c|c|c|}
\hline \multirow[b]{2}{*}{ Groups } & \multirow{2}{*}{ Time point } & \multirow{2}{*}{$\mathbf{N}$} & \multicolumn{2}{|c|}{$\geq 0.5 \mathrm{EU} / \mathrm{mL}$} & \multirow{2}{*}{$\begin{array}{c}\text { GMT } \\
\text { value }(95 \% \mathrm{CI})\end{array}$} \\
\hline & & & $\mathbf{n}$ & $\%(95 \% \mathrm{CI})$ & \\
\hline \multirow{5}{*}{ RTS,S/AS01 vaccine } & Screening & 81 & 16 & $19 \cdot 8(11 \cdot 7-30 \cdot 1)$ & $0 \cdot 3(0 \cdot 3-0 \cdot 4)$ \\
\hline & $1 \mathrm{M}$ post-dose 3 & 79 & 79 & $100(95 \cdot 4-100)$ & $329 \cdot 2(260 \cdot 6-415 \cdot 8)$ \\
\hline & $12 \mathrm{M}$ post-dose 3 & 73 & 72 & $98 \cdot 6(92 \cdot 6-100)$ & $18 \cdot 4(13 \cdot 3-25 \cdot 5)$ \\
\hline & Screening & 73 & 13 & $17 \cdot 8(9 \cdot 8-28 \cdot 5)$ & $0 \cdot 3(0 \cdot 3-0 \cdot 4)$ \\
\hline & $1 \mathrm{M}$ post-dose 3 & 71 & 9 & $12 \cdot 7(6 \cdot 0-22 \cdot 7)$ & $0 \cdot 3(0 \cdot 3-0 \cdot 3)$ \\
\hline Rabies vaccine & $12 \mathrm{M}$ post-dose 3 & 67 & 6 & $9 \cdot 0(3 \cdot 4-18 \cdot 5)$ & $0 \cdot 3(0 \cdot 3-0 \cdot 3)$ \\
\hline
\end{tabular}

$\mathrm{ATP}=$ according-to-protocol. EU=enzyme-linked immunosorbent assay unit. GMT=geometric mean antibody titre. $\mathrm{N}=$ number of children with available results. $\mathrm{n} / \%=$ number/percentage of children with titre equal to or above $0.5 \mathrm{EU} / \mathrm{mL}$. $\mathrm{CI}=$ confidence interval. $\mathrm{M}=$ month(s). 


\section{FIGURE LEGENDS}

\section{Figure 1. CONSORT diagram}

$\mathrm{ATP}=$ according to protocol. $\mathrm{SAE}=$ serious adverse event.

Figure 2: Overall/dose incidence of solicited and grade 3 solicited adverse events reported during the 7-day post-vaccination periods (intention-to-treat population)

$\mathrm{N}=$ number of administered doses. Grade $3=$ crying when limb was moved/limb was spontaneously painful (pain), diameter $>20 \mathrm{~mm}$ (redness, swelling), preventing everyday activity (drowsiness, irritability), crying inconsolably (irritability), not eating at all (loss of appetite), axillary temperature $>39.0^{\circ} \mathrm{C}$ (fever). Any fever $=$ axillary temperature $\geq 37 \cdot 5^{\circ} \mathrm{C}$. Error bars represent $95 \%$ confidence intervals. 


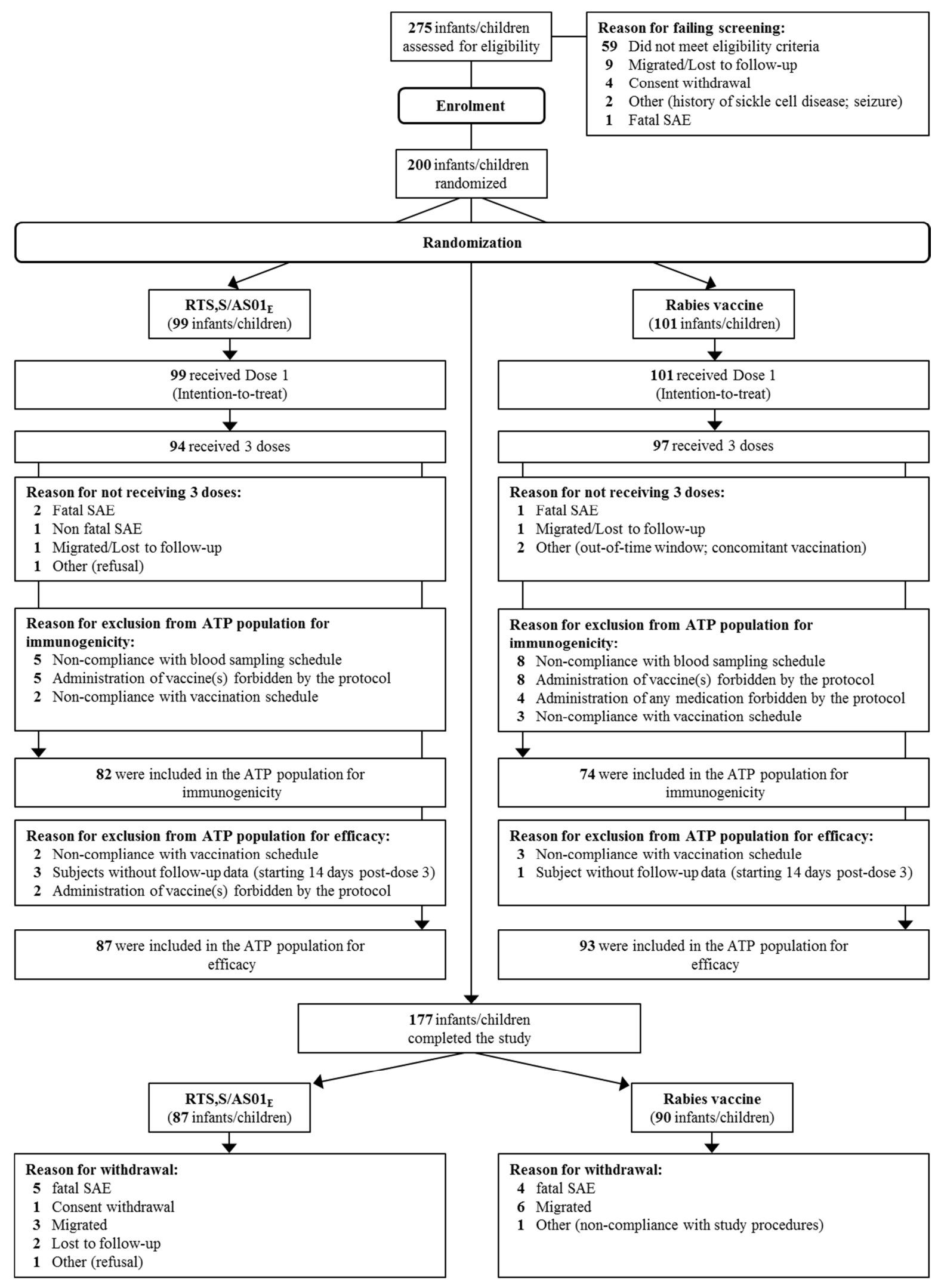




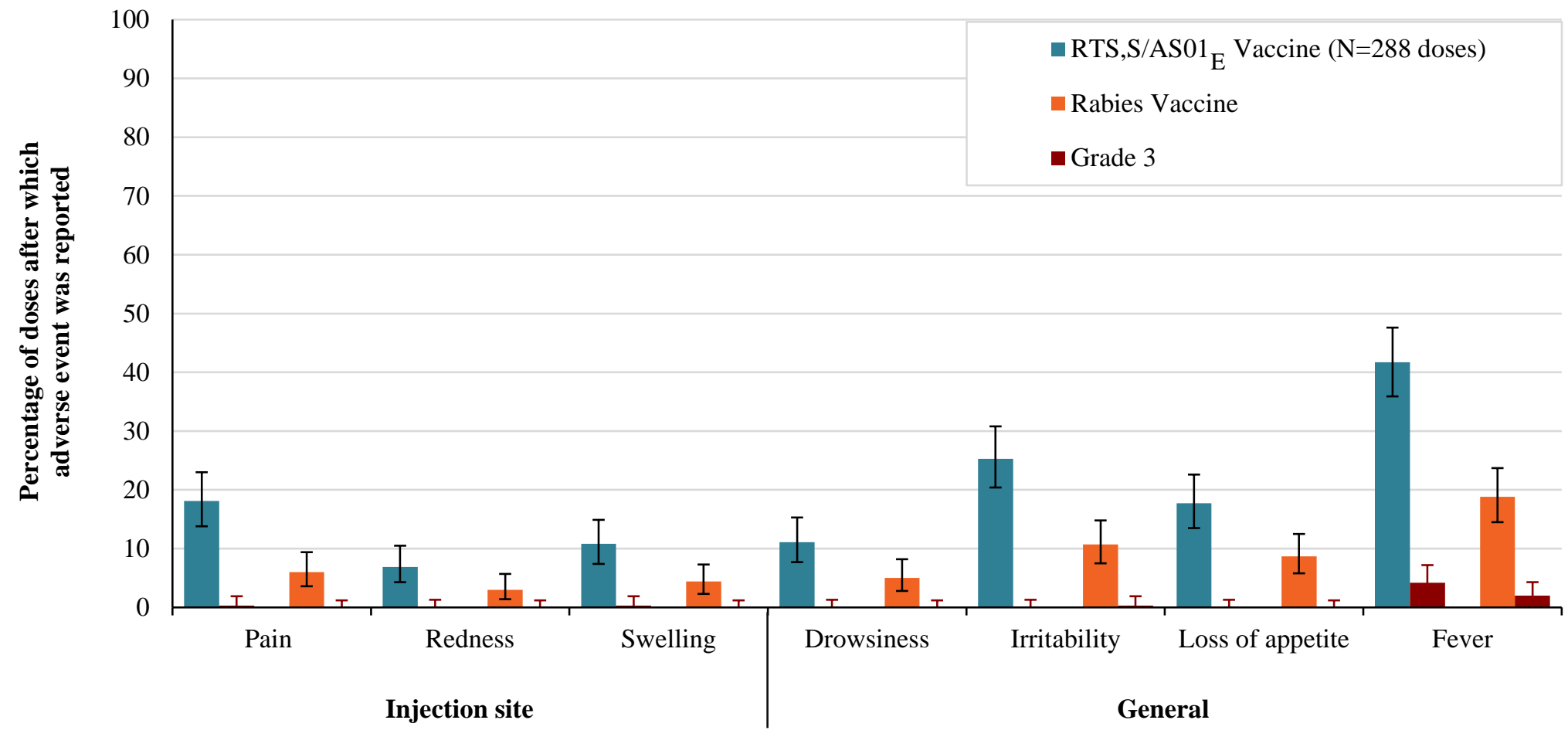

